\title{
Review: prenatal exposure to cocaine does not independently affect physical growth, cognition, or language skills
} Frank DA, Augustyn M, Grant Knight Wet al. Growth, development, and behavior in early childhood following prenatal
cocaine exposure: a systematic review.JAMA 2001 Mar 28;285:1613-25.

QUESTION: Do children exposed to cocaine in utero have worse outcomes than unexposed children in the domains of physical growth; cognition; language skills; motor skills; and behaviour, attention, affect, and neurophysiology?

\section{Data sources}

Studies were identified by searching Medline and PsycINFO (1984-2000) using the terms cocaine, crack, pregnancy, prenatal exposure, delayed effects, children, and related disorders.

\section{Study selection}

English language peer reviewed published studies were selected if the patients were prospectively recruited, the outcome assessors were blinded to cocaine exposure status, and the cocaine exposed cohort did not include a substantial proportion of children also exposed in utero to opiates, amphetamines, or phencyclidine; or whose mothers were HIV positive.

\section{Data extraction}

Data were extracted on number of exposed and unexposed children; concentrations of cocaine exposure; and pregnancy exposure to tobacco, alcohol, and marijuana.

\section{Main results}

36 studies were included. Of 7 studies that examined the effect of prenatal cocaine exposure on physical growth, no effect was shown after controlling for exposure to tobacco and alcohol. 9 studies examined cognitive development in infants: 5 studies showed no effect; 4 studies showed an association that could not be separated from the effects of maternal alcohol or marijuana use, other risk factors, or design flaws relating to lack of adjustment or post hoc comparisons. 6 studies that evaluated cognitive development in children 3-6 years of age showed no effect when alcohol, marijuana, or tobacco use were controlled for (3 studies); 3 studies showed no effect on intellectual quotient, cognitive test scores, or academic skills. 3 studies that assessed language skills among toddlers showed no association with prenatal cocaine exposure. 6 studies assessed motor skills: 3 studies showed less optimal scores at $\leqslant 7$ months of age. 1 study showed tobacco use, not cocaine, was the main predictor of muscle tone abnormalities at 6 weeks of age. For the outcomes of behaviour, attention, affect, and neurophysiology, 2 studies of 7 showed less optimal behaviour, but the evaluation tool was an unvalidated teacher report form. 4 studies showed decreased affective expression in children $<2$ years of age.

\section{Conclusions}

Prenatal cocaine exposure does not have an independent effect on children in the domains of physical growth, cognition, or language skills. Some evidence indicates less optimal motor skills at $\leqslant 7$ months of age and decreased expression of affect at $<2$ years of age.

\section{COMMENTARY}

This systematic review by Frank et al of the outcomes, in early childhood, of those children born to a cocaine using mother is of great importance. Since it only considers studies of those samples prospectively recruited in the perinatal period, and excludes studies of children substantially exposed, in utero, to opiates, amphetamines, phencyclidine, or maternal HIV infection, it allows for a clearer consideration of the actual effect of prenatal cocaine exposure. This distinction is of utmost importance when decisions about the risk to a child are focused particularly on cocaine use during pregnancy as a primary risk factor.

The authors have used rigorous methodology to select the studies and present a useful table of the studies included while correctly emphasising that the knowledge base on the effects of prenatal cocaine exposure remains limited. Similarly, they emphasise the multiple risks to which such cohorts of children are exposed pharmacologically, psychologically, and socially. Additionally, the authors make clear that despite a systematic review on prospectively recruited cohorts, potential bias issues remain. Their findings, however, that no consistent negative association existed between prenatal cocaine exposure and physical growth, developmental test scores, or receptive or expressive language and that there may be an effect on motor scores up to the age of 7 months but not thereafter, should be of great interest to practitioners. These findings should also influence further rigorous studies of cocaine exposure in children born to cocaine using mothers. Furthermore, practitioners are correctly reminded that prenatal exposure to tobacco, alcohol, and poor environment needs to be actively addressed, together with cocaine use, in the pregnant drug user.

Judith Myles, MB, BCH, FRCPsych Blackberry Hill Hospital, Bristol, UK
Source of funding: Drug Abuse.

For correspondence: Dr D A Frank, Boston Medical Center, Growth and Development Programs, 820 Harrison Avenue, FGH Building, Third Floor, Boston, $M A$ 02118-2393, USA $\mathrm{Fax}+1617414$ 7047. 